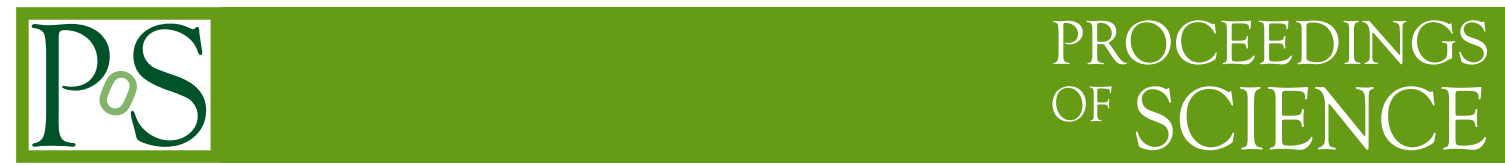

\title{
Early stage thermalization via instabilities
}

\author{
Stanisław Mrówczyński ${ }^{*}$ \\ Institute of Physics, Świętokrzyska Academy, ul. Świętokrzyska 15, PL - 25-406 Kielce, Poland \\ and Soltan Institute for Nuclear Studies, ul. Hoza 69, PL - 00-681 Warsaw, Poland \\ E-mail: mrowefuw.edu.pl
}

\begin{abstract}
Due to anisotropic momentum distributions the parton system produced at the early stage of relativistic heavy-ion collisions is unstable with respect to the magnetic plasma modes. The instabilities isotropize the system and thus speed up the process of its equilibration. The whole scenario of the instabilities driven isotropization is reviewed.
\end{abstract}

Critical Point and Onset of Deconfinement

July 3-6 2006

Florence, Italy

\footnotetext{
* Speaker.

${ }^{\dagger}$ This is the updated version of the review [1].
} 


\section{Introduction}

The matter created in relativistic heavy-ion collisions manifests a strongly collective hydrodynamic behaviour [2] which is particularly evident in studies of the so-called elliptic flow [3]. A hydrodynamic description requires, strictly speaking, a local thermal equilibrium and experimental data on the particle spectra and elliptic flow suggest, when analysed within the hydrodynamic model, that an equilibration time of the parton ${ }^{1}$ system produced at the collision early stage is as short as $0.6 \mathrm{fm} / c$ [4]. Such a fast equilibration can be explained assuming that the quark-gluon plasma is strongly coupled [5]. However, it is not excluded that due to the high-energy density at the early stage of the collision, when the elliptic flow is generated [6], the plasma is relatively weakly coupled because of asymptotic freedom. Thus, the question arises whether the weakly interacting plasma can be equilibrated within $1 \mathrm{fm} / c$.

Models that assume that parton-parton collisions are responsible for the thermalization of weakly coupled plasma provide a significantly longer equilibration time. The calculations performed within the 'bottom-up' thermalization scenario [7], where the binary and $2 \leftrightarrow 3$ processes are taken into account, give an equilibration time of at least $2.6 \mathrm{fm} / c$ [8]. To thermalize the system one needs either a few hard collisions of momentum transfer of order of the characteristic parton momentum ${ }^{2}$, which is denoted here as $T$ (as the temperature of equilibrium system), or many collisions of smaller transfer. As discussed in e.g. [9], the inverse equilibration time is of order $g^{4} \ln (1 / g) T$ (with $g$ being the QCD coupling constant) when the binary collisions are responsible for the system's thermalization. However, the equilibration is speeded up by instabilities generated in an anisotropic quark-gluon plasma $[10,11]$, as growth of the unstable modes is associated with the system's isotropization. The characteristic inverse time of instability development is roughly of order $g T$ for a sufficiently anisotropic momentum distribution $[10,11,12,13,14,15]$. Thus, the instabilities are much 'faster' than the collisions in the weak coupling regime. Recent numerical simulation [16] shows that the instabilities driven isotropization is indeed very efficient.

The isotropization should be clearly distinguished from the equilibration. The instabilities driven isotropization is a mean-field reversible phenomenon which is not accompanied with entropy production $[10,16]$. Therefore, the collisions, which are responsible for the dissipation, are needed to reach the equilibrium state of maximal entropy. The instabilities contribute to the equilibration indirectly, shaping the parton momenta distribution. And recently it has been argued [11] that the hydrodynamic collective behaviour does not actually require local thermodynamic equilibrium but a merely isotropic momentum distribution of liquid components. Thus, the above mentioned estimate of $0.6 \mathrm{fm} / c$ [4] rather applies to the isotropization than to the equilibration.

My aim here is to review the whole scenario of instabilities driven isotropization and the article is organized as follows. I start with a brief presentation of numerous efforts to understand the equilibration process of the quark-gluon plasma which have been undertaken over last two decades. In Sec. 3 various plasma instabilities are considered and the magnetic Weibel modes are argued to be relevant for the quark-gluon plasma produced in relativistic heavy-ion collisions. In

\footnotetext{
${ }^{1}$ The term 'parton' is used to denote a quasiparticle fermionic (quark) or bosonic (gluon) excitation of the quarkgluon plasma.

${ }^{2}$ Although anisotropic systems are considered, the characteristic momentum in all directions is assumed to be of the same order.
} 
Sec. 4 I discuss how the unstable modes are initiated while in Sec. 5 the mechanism of unstable mode growth is explained in terms of elementary physics. Sec. 6 is devoted to solutions of the dispersion equation which provide dispersion relations of the unstable modes. In Sec. 7 it is explained why the instabilities isotropize the system. A phenomenon of spontaneous abelianization of the system's configuration is considered in the same section. The two next sections contain more formal material. The Hard Loop effective action of anisotropic plasma is presented in Sec. 8 while Sec. 9 deals with the equations of motion which are used to study temporal evolution of anisotropic plasma. Results of recent numerical simulations of the plasma evolution are presented in Sec. 10. The review is closed with a brief discussion on possible signals of the instabilities and on desired improvements of theoretical approaches to the unstable quark-gluon plasma.

Throughout the article there are used the natural units with $\hbar=c=k_{\mathrm{B}}=1$; the metric convention is $(1,-1,-1,-1)$; the coupling constant $\alpha_{s} \equiv g^{2} / 4 \pi$ is assumed to be small; quarks and gluons are massless.

\section{Equilibration of the Quark-Gluon Plasma}

To present the scenario of instabilities driven isotropization in a broader context, I start with a brief review of numerous attempts to understand the equilibration processes of the quark-gluon. The problem was posed over twenty years ago when the real prospects to create the quark-gluon plasma in terrestrial experiments appeared. Already in the early papers published in the eighties $[17,18,19,20,21,22,23]$, main directions of further studies were drawn. The space-time structure of ultrarelativistic heavy-ion collisions was found [20] to provide an estimate of the system's temperature and the lower bound of the thermalization time. The Boltzmann equation in the Relaxation Time Approximation [17] and the Fokker-Planck equation [18] were used to follow the equilibration process. The Schwinger mechanism of particle production was included in kinetic theory treatment of the thermalization $[19,23]$ and the pure perturbative mechanism was analysed as well [22]. The equilibration was also studied within the Monte Carlo parton cascade model [21] which, however, took into account only binary parton-parton collisions.

These lines of research were continued in the next decade. The parton cascade approach was greatly improved [24] by, in particular, including the gluon radiation in the initial and final states of parton-parton interactions. The radiation proved to be very important for the equilibration process $[25,26]$. These detailed numerical studies are summarized in the review [27]. Another perturbative parton cascade approach combined with the string phenomenology for non-perturbative interactions is presented in [28]. The analytical studies of the thermalization were continued in $[29,30,31,32,33]$, see also [28], where, in particular, the gluons were convincingly shown to equilibrate much faster than the quarks, the free streaming and the role of infrared cut-offs in the parton-parton cross sections were elucidated. Much efforts were invested in the studies of multiparticle processes $[34,35,36,37]$ which were already implemented in the parton cascade type models [27, 28]. The inelastic process $2 \leftrightarrow 3$ attracted a lot of attention. Although it is of higher order in $\alpha_{s}$, it is responsible for the parton number equilibration and it dominates the entropy production $[35,36,37]$.

There are two very recent transport theory approaches to the equilibration problem based on big numerical codes where the role of the multi-particle processes is emphasized [38, 39]. The 
authors of [38] include particle production and absorption via the process $2 \leftrightarrow 3$ while the threeparticle collisions $3 \leftrightarrow 3$ are studied in [39]. Within both approaches the equilibration is claimed to be significantly speeded-up when compared to the equilibration driven by the binary collisions. However, the interaction rates of multi-particle processes are known to suffer from severe divergences, and thus, the actual role of the multi-particle interactions crucially depends on how the rates are defined, computed and regularized.

The observation that the multi-particle interaction rates are sometimes divergent was actually used to explain the very fast equilibration of the quark-gluon system. The so-called collinear divergences of the gluon multiplication process $2 \leftrightarrow 3$ cancel in the equilibrium. If the cancellation does not occur in the non-equilibrium systems, as argued in [40], the equilibration, which is driven by very large - formally divergent - interaction rates, is extremely fast even in the weakly coupled plasma [40].

The thermalization of the quark-gluon plasma was also discussed from a very different point of view where the equilibration is not due to the inter-parton collisions but due to the chaotic dynamics of the non-Abelian classical fields (coupled or not to the classical coloured particles) [41, 42], see also a very recent paper [43]. Then, the equilibration time is controlled by the maximal Lyapunov exponent.

At the turn of the millennium, when a large volume of experimental data from the RHIC started to flow, understanding of the equilibration process became a burning issue as the data favoured a very short equilibration mentioned in the Introduction. Within the concept of strongly coupled quark-gluon plasma, the problem is trivially solved, as the strongly interacting system is indeed equilibrated very fast. However, it is still an open issue whether the plasma at the collision early stage is indeed strongly coupled.

A novel development concerned a treatment of the initial state of the parton system which evolves towards equilibrium. In the papers mentioned above, one usually assumed that the initial partons are produced due to the (semi-)hard interactions of partons of the incident nuclei. Thus, jets and minijets form such an initial state which can be parametrized in several ways [44]. Recent studies of the equilibration problem which adopt the minijet initial conditions are presented in $[45,46,47]$.

In the already mentioned 'bottom-up' thermalization scenario [7], the initial state was assumed to be shaped by the QCD saturation mechanism. Then, the initial state is dominated by the small $x$ gluons of transverse momentum of order $Q_{s}$ which is the saturation scale. These gluons are freed from the incoming nuclei after a time $Q_{s}^{-1}$. Weak coupling techniques are applicable as $Q_{s}$ is expected to be much smaller than $\Lambda_{\mathrm{QCD}}$ at sufficiently high collision energies. The saturation mechanism is incorporated in the effective field approach known as the Colour Glass Condensate [48] where the small $x$ partons of large occupation numbers are treated as classical Yang-Mills fields. Hard modes of the classical fields play the role of particles here. The equilibration processes with the minijet and saturation initial states were compared to each other in [49].

The 'bottom-up' thermalization scenario [7], where not only binary collisions but the processes $2 \leftrightarrow 3$ are included, takes into account the system's expansion. The equilibration processes splits into several stages parametrically characterized by $\alpha_{s}^{n} Q_{s}^{-1}$ where $n$ is a fractional power. The thermalization time is of order $\alpha_{s}^{-13 / 5} Q_{s}^{-1}$. However, as stressed in the Introduction, the collisional isotropization is apparently too slow to comply with the experimental data. The calculations per- 
formed within the 'bottom-up' scenario [7] were criticized [14] for treating the parton momentum distribution as isotropic, and thus, ignoring the instabilities which actually speed up the equilibration process. Recently, an influence of the instabilities on the 'bottom-up' time scales has been discussed in [50]. It has been also argued [51] that a somewhat modified scenario remains valid for a sufficiently late stage of the equilibration process when the instabilities are no longer operative.

At the end I mention rather unconventional approaches to the fast equilibration problem. It was argued in $[52,53,54]$ that the momentum distribution of partons is of the equilibrium form just after the production process. Thus, the very process of particle production leads to the equilibrium state without any secondary interactions. The authors of $[52,53]$ refer to the Schwinger mechanism of particle's production due to the strong chromoelectric field. The transverse momentum but not longitudinal one is claimed to be 'equilibrated' in this way [52, 53]. The key ingredient of the approach [54], where the longitudinal momentum is also thermal, is the Hawking-Unruh effect: an observer moving with an acceleration $a$ experiences the influence of a thermal bath with an effective temperature $a / 2 \pi$, similar to the one present in the vicinity of a black hole horizon. The idea behind the approaches $[52,53,54]$ is elegant and universal - it can be applied not only to nucleus-nucleus but to hadron-hadron or even to $e^{+} e^{-}$collisions - but it cannot explain how the equilibrium state is maintained when the parton's free streaming drives the system out of equilibrium. Secondary interactions are then certainly needed.

Finally, I note a very interesting 'no-go' theorem [55, 56], which states that the perturbative thermalization is impossible, as any Feynman diagram of any order leads in the long time limit to the time scaling of the energy density corresponding to the free streaming, not to the Bjorken hydrodynamics. However, it is not quite clear whether the theorem applies to the relativistic heavyion collisions as the equilibrium state of matter produced in the collisions is presumably only a transient state which changes into free streaming at the late times of the system's evolution.

\section{Relevant Plasma Instabilities}

The electron-ion plasma is known to experience a large variety of instabilities [57]. Those caused by coordinate space inhomogeneities, in particular by the system's boundaries, are usually called hydrodynamic instabilities, while those due to non-equilibrium momentum distribution of plasma particles are called kinetic instabilities. Hardly anything is known about hydrodynamic instabilities of the quark-gluon plasma, and I will not speculate about their possible role in the system's dynamics. The kinetic instabilities are initiated either by the charge or current fluctuations. In the first case, the electric field (E) is longitudinal ( $\mathbf{E} \| \mathbf{k}$, where $\mathbf{k}$ is the wave vector), while in the second case the field is transverse $(\mathbf{E} \perp \mathbf{k})$. For this reason, the kinetic instabilities caused by the charge fluctuations are usually called longitudinal while those caused by the current fluctuations are called transverse. Since the electric field plays a crucial role in the longitudinal mode generation, the longitudinal instabilities are also called electric while the transverse ones are called magnetic. In the non-relativistic plasma the electric instabilities are usually much more important than the magnetic ones, as the magnetic effects are suppressed by the factor $v^{2} / c^{2}$ where $v$ is the particle's velocity. In the relativistic plasma both types of instabilities are of similar strength. The electric instabilities occur when the momentum distribution of plasma particles has more than one 
maximum, as in the two-stream system. A sufficient condition for the magnetic instabilities is, as discussed in Sec. 6, anisotropy of the momentum distribution.

Soon after the concept of quark-gluon plasma had been established, the existence of the colour kinetic instabilities, fully analogous to those known in the electrodynamic plasma, was suggested $[58,59,60,61,62,63,64]$. In these early papers, however, there was considered a two-stream system, or more generally, a momentum distribution with more than one maximum. While such a distribution is common in the electron-ion plasma, it is rather irrelevant for the quark-gluon plasma produced in relativistic heavy-ion collisions where the global as well as local momentum distribution is expected to monotonously decrease in every direction from the maximum. The electric instabilities are absent in such a system but, as demonstrated in $[65,10]$, a magnetic unstable mode known as the filamentation or Weibel instability [66] is possible. The filamentaion instability was shown $[65,10]$ to be relevant for the quark-gluon plasma produced in relativistic heavy-ion collisions as the characteristic time of instability growth is shorter or at least comparable to other time scales of the parton system evolution. And the instabilities - usually not one but several modes are generated - drive the system towards isotropy, thus speeding up its equilibration. In the following sections a whole scenario of the instabilities driven equilibration is reviewed.

\section{Seeds of filamentation}

Let me start with a few remarks on degrees of freedom of the quark-gluon plasma. Various problems will be repeatedly discussed in terms of classical fields and particles which are only approximate notions in the quark-gluon plasma being a system of relativistic quantum fields. However, collective excitations, which are bosonic and highly populated, can be treated as classical fields while bosonic or fermionic excitations, with the energy determined by the excitation momentum (due to the dispersion relation), can be treated as (quasi-)particles. In the weakly coupled quark-gluon plasma in equilibrium, an excitation is called hard when its momentum is of order $T$, which is the system's temperature, and it is called soft when its momentum is of order $g T$. Within the Hard Loop dynamics, the hard excitations can be treated as particles while the gluonic soft excitations as classical fields [67]. It is expected that a similar treatment is possible in the non-equilibrium plasma as well. Thus, the terms partons, quarks, gluons, particles will be used to denote quasiparticle hard excitations. The classical chromodynamic field will represent gluonic soft collective excitations.

After the introductory remarks, let me discuss how the unstable transverse modes are initiated. For this purpose I consider a parton system which is homogeneous but the parton momentum distribution is not of the equilibrium form, it is not isotropic. The system is on average locally colourless but colour fluctuations are possible. Therefore, $\left\langle\mu_{a}^{\mu}(x)\right\rangle=0$ where $j_{a}^{\mu}(x)$ is a local colour four-current in the adjoint representation of $\mathrm{SU}\left(N_{c}\right)$ gauge group with $\mu=0,1,2,3$ and $a=1,2, \ldots,\left(N_{c}^{2}-1\right)$ being the Lorentz and colour index, respectively; $x=(t, \mathbf{x})$ denotes a fourposition in coordinate space.

Since I assume that the quark-gluon plasma is weakly coupled, the non-interacting gas of quarks, antiquarks and gluons can be treated as a first approximation. As discussed in detail in 
[68], the current correlator for a classical system of non-interacting massless partons is

$$
M_{a b}^{\mu v}(t, \mathbf{x}) \stackrel{\text { def }}{=}\left\langle j_{a}^{\mu}\left(t_{1}, \mathbf{x}_{1}\right) j_{b}^{v}\left(t_{2}, \mathbf{x}_{2}\right)\right\rangle=\frac{1}{8} g^{2} \delta^{a b} \int \frac{d^{3} p}{(2 \pi)^{3}} \frac{p^{\mu} p^{v}}{\mathbf{p}^{2}} f(\mathbf{p}) \delta^{(3)}(\mathbf{x}-\mathbf{v} t),
$$

where $\mathbf{v} \equiv \mathbf{p} /|\mathbf{p}|,(t, \mathbf{x}) \equiv\left(t_{2}-t_{1}, \mathbf{x}_{2}-\mathbf{x}_{1}\right)$ and the effective parton distribution function $f(\mathbf{p})$ equals $n(\mathbf{p})+\bar{n}(\mathbf{p})+2 N_{c} n_{g}(\mathbf{p}) ; n(\mathbf{p}), \bar{n}(\mathbf{p})$ and $n_{g}(\mathbf{p})$ give the average colourless distribution function of quarks $Q^{i j}(\mathbf{p}, x)=\delta^{i j} n(\mathbf{p})$, antiquarks $\bar{Q}^{i j}(\mathbf{p}, x)=\delta^{i j} \bar{n}(\mathbf{p})$, and gluons $G^{a b}(\mathbf{p}, x)=\delta^{a b} n_{g}(\mathbf{p})$. The distribution function of (anti-)quarks and gluons are matrices belonging to the fundamental and ajoint representation, respectively, of the $\mathrm{SU}\left(N_{c}\right)$ gauge group. Therefore, $i, j=1,2, \ldots, N_{c}$ and $a, b=1,2, \ldots,\left(N_{c}^{2}-1\right)$.

Due to the average space-time homogeneity, the correlation tensor (4.1) depends only on the difference $\left(t_{2}-t_{1}, \mathbf{x}_{2}-\mathbf{x}_{1}\right)$. The space-time points $\left(t_{1}, \mathbf{x}_{1}\right)$ and $\left(t_{2}, \mathbf{x}_{2}\right)$ are correlated in the system of non-interacting particles if a particle travels from $\left(t_{1}, \mathbf{x}_{1}\right)$ to $\left(t_{2}, \mathbf{x}_{2}\right)$. For this reason the delta $\delta^{(3)}(\mathbf{x}-\mathbf{v} t)$ is present in the formula (4.1). The momentum integral of the distribution function simply represents the summation over particles. The fluctuation spectrum is found as a Fourier transform of the tensor (4.1) i.e.

$$
M_{a b}^{\mu v}(\omega, \mathbf{k})=\frac{1}{8} g^{2} \delta^{a b} \int \frac{d^{3} p}{(2 \pi)^{3}} \frac{p^{\mu} p^{v}}{\mathbf{p}^{2}} f(\mathbf{p}) 2 \pi \delta(\omega-\mathbf{k v}) .
$$

To compute the fluctuation spectrum, the parton momentum distribution has to be specified. Such calculations with two forms of the momentum distribution are presented in [68]. Here I only qualitatively discuss Eqs. $(4.1,4.2)$, assuming that the parton momentum distribution is anisotropic.

In heavy-ion collisions, the anisotropy is a generic feature of the parton momentum distribution in a local rest frame. After the first collisions, when the partons are released from the incoming nucleons, the momentum distribution is strongly elongated along the beam - it is of the prolate shape with the average transverse momentum being much smaller than the average longitudinal one. Due to the free streaming, it evolves in the local rest frame to the distribution which is squeezed along the beam - it is of the oblate shape with the average transverse momentum being much larger than the average longitudinal one. In most cases, I assume that the distribution is elongated along the $z$ axis but my considerations remain valid for the distribution, which is squeezed along the $z$ axis, but the axes should be relabeled.

With the momentum distribution elongated in the $z$ direction, Eqs. $(4.1,4.2)$ clearly show that the correlator $M^{z z}$ is larger than $M^{x x}$ or $M^{y y}$. It is also clear that $M^{z z}$ is the largest when the wave vector $\mathbf{k}$ is along the direction of the momentum deficit. Then, the delta function $\delta(\omega-\mathbf{k v})$ does not much constrain the integral in Eq. (4.2). Since the momentum distribution is elongated in the $z$ direction, the current fluctuations are the largest when the wave vector $\mathbf{k}$ is the $x-y$ plane. Thus, I conclude that some fluctuations in the anisotropic system are large, much larger than in the isotropic one. An anisotropic system has a natural tendency to split into the current filaments parallel to the direction of the momentum surplus. These currents are seeds of the filamentation instability. 


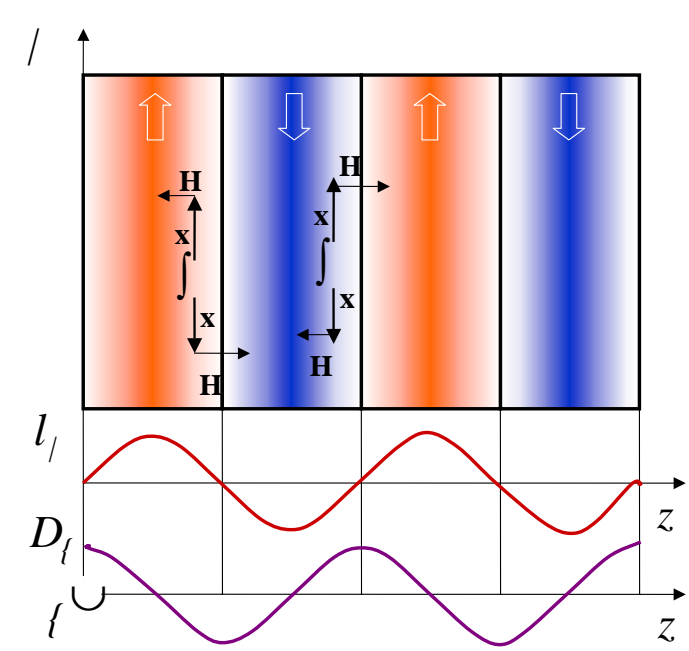

Figure 1: The mechanism of filamentation instability, see text for a description.

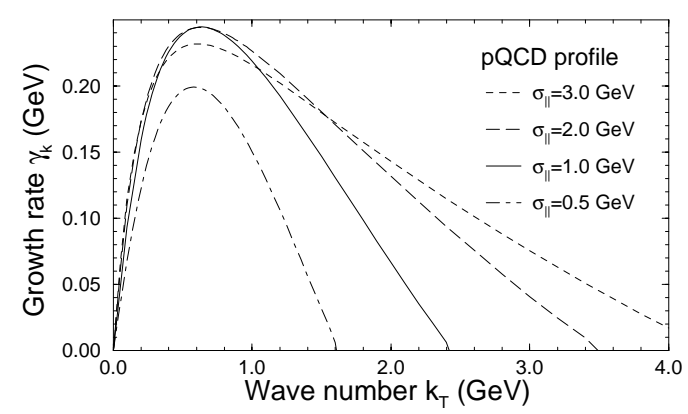

Figure 2: The growth rate of the unstable mode as a function of the wave vector $\mathrm{k}=\left(k_{\perp}, 0,0\right)$ for $\sigma_{\perp}=0.3 \mathrm{GeV}$ and 4 values of the parameter $\sigma_{\|}$ which controls system's anisotropy. The figure is taken from [12].

\section{Mechanism of filamentation}

Let me now explain in terms of elementary physics why the fluctuating currents, which flow in the direction of the momentum surplus, can grow in time. To simplify the discussion, which follows [68], I consider an electromagnetic anisotropic system. The form of the fluctuating current is chosen to be

$$
\mathbf{j}(x)=j \hat{\mathbf{e}}_{z} \cos \left(k_{x} x\right),
$$

where $\hat{\mathbf{e}}_{z}$ is the unit vector in the $z$ direction. As seen in Eq. (5.1), there are current filaments of the thickness $\pi /\left|k_{x}\right|$ with the current flowing in the opposite directions in the neighbouring filaments. The magnetic field generated by the current (5.1) is given as

$$
\mathbf{B}(x)=\frac{j}{k_{x}} \hat{\mathbf{e}}_{y} \sin \left(k_{x} x\right),
$$

and the Lorentz force acting on the partons, which fly along the $z$ direction, equals

$$
\mathbf{F}(x)=q \mathbf{v} \times \mathbf{B}(x)=-q v_{z} \frac{j}{k_{x}} \hat{\mathbf{e}}_{x} \sin \left(k_{x} x\right),
$$

where $q$ is the electric charge. One observes, see Fig. 1, that the force distributes the partons in such a way that those, which positively contribute to the current in a given filament, are focused in the filament centre while those, which negatively contribute, are moved to the neighbouring one. Thus, the initial current is growing and the magnetic field generated by this current is growing as well. The instability is driven by the the energy transferred from the particles to fields. More specifically, the kinetic energy related to a motion along the direction of the momentum surplus is used to generate the magnetic field. The mechanism of Weibel instability is explained somewhat differently in [14]. 


\section{Dispersion equation}

The Fourier transformed chromodynamic field $A^{\mu}(k)$ satisfies the equation of motion as

$$
\left[k^{2} g^{\mu v}-k^{\mu} k^{v}-\Pi^{\mu v}(k)\right] A_{v}(k)=0,
$$

where $k \equiv(\omega, \mathbf{k})$ and $\Pi^{\mu v}(k)$ is the polarization tensor or gluon self-energy which is discussed later on. Since the tensor is proportional to a unit matrix in the colour space, the colour indices are dropped here. A general plasmon dispersion equation is of the form

$$
\operatorname{det}\left[k^{2} g^{\mu v}-k^{\mu} k^{v}-\Pi^{\mu v}(k)\right]=0 .
$$

Equivalently, the dispersion relations are given by the positions of poles of the effective gluon propagator. Due to the transversality of $\Pi^{\mu v}(k)\left(k_{\mu} \Pi^{\mu v}(k)=k_{v} \Pi^{\mu v}(k)=0\right)$ not all components of $\Pi^{\mu v}(k)$ are independent from each other, and consequently the dispersion equation (6.2), which involves a determinant of a $4 \times 4$ matrix, can be simplified to the determinant of a $3 \times 3$ matrix. For this purpose I introduce the colour permittivity tensor $\varepsilon^{m}(k)$ where the indices $l, m, n=1,2,3$ label three-vector and tensor components. Because of the relation

$$
\varepsilon^{l m}(k) E^{l}(k) E^{m}(k)=\Pi^{\mu v}(k) A_{\mu}(k) A_{v}(k),
$$

where $\mathbf{E}$ is the chromoelectric vector, the permittivity can be expressed through the polarization tensor as

$$
\varepsilon^{l m}(k)=\delta^{l m}+\frac{1}{\omega^{2}} \Pi^{l m}(k) .
$$

Then, the dispersion equation gets the form

$$
\operatorname{det}\left[\mathbf{k}^{2} \delta^{l m}-k^{l} k^{m}-\omega^{2} \varepsilon^{l m}(k)\right]=0 .
$$

The relationship between Eq. (6.2) and Eq. (6.3) is most easily seen in the Coulomb gauge when $A^{0}=0$ and $\mathbf{k} \cdot \mathbf{A}(k)=0$. Then, $\mathbf{E}=i \omega \mathbf{A}$ and Eq. (6.1) is immediately transformed into an equation of motion of $\mathbf{E}(k)$ which further provides the dispersion equation (6.3).

The dynamical information is contained in the polarization tensor $\Pi^{\mu v}(k)$ given by Eq. (8.2) or, equivalently, in the permittivity tensor $\varepsilon^{m}(k)$ which can be derived either within the transport theory or diagrammatically [69]. The result is

$$
\varepsilon^{n m}(\omega, \mathbf{k})=\delta^{n m}+\frac{g^{2}}{2 \omega} \int \frac{d^{3} p}{(2 \pi)^{3}} \frac{v^{n}}{\omega-\mathbf{k v}+i 0^{+}} \frac{\partial f(\mathbf{p})}{\partial p^{l}}\left[\left(1-\frac{\mathbf{k v}}{\omega}\right) \delta^{l m}+\frac{k^{l} v^{m}}{\omega}\right] .
$$

As already mentioned, the colour indices are suppressed here.

Substituting the permittivity (6.4) into Eq. (6.3), one fully specifies the dispersion equation (6.3) which provides a spectrum of quasi-particle bosonic excitations. A solution $\omega(\mathbf{k})$ of Eq. (6.3) is called stable when $\operatorname{Im} \omega \leq 0$ and unstable when $\operatorname{Im} \omega>0$. In the first case the amplitude is constant or it exponentially decreases in time while in the second one there is an exponential growth of the amplitude. In practice, it appears difficult to find solutions of Eq. (6.3) because of the rather 
complicated structure of the tensor (6.4). However, the problem simplifies as we are interested in specific modes which are expected to be unstable. Namely, we look for solutions corresponding to the fluctuating current in the direction of the momentum surplus and the wave vector perpendicular to it.

As previously, the momentum distribution is assumed to be elongated in the $z$ direction, and consequently the fluctuating current also flows in this direction. The magnetic field has a nonvanishing component along the $y$ direction and the electric field in the $z$ direction. Finally, the wave vector is parallel to the axis $x$, see Fig. 1. It is also assumed that the momentum distribution obeys the mirror symmetry $f(-\mathbf{p})=f(\mathbf{p})$, and then the permittivity tensor has only non-vanishing diagonal components. Taking into account all these conditions, one simplifies the dispersion equation (6.3) to the form

$$
H(\omega) \equiv k_{x}^{2}-\omega^{2} \varepsilon^{z z}\left(\omega, k_{x}\right)=0,
$$

where only one diagonal component of the dielectric tensor enters.

It appears that the existence of unstable solutions of Eq. (6.5) can be proved without solving it. The so-called Penrose criterion [57], which follows from analytic properties of the permittivity as a function of $\omega$, states that the dispersion equation $H(\omega)=0$ has unstable solutions if $H(\omega=0)<0$. The Penrose criterion was applied to the equation (6.5) in [10] but a more general discussion of the instability condition is presented in [14]. Not entering into details, there exist unstable modes if the momentum distribution averaged (with a proper weight) over momentum length is anisotropic.

To solve the dispersion equation (6.5), the parton momentum distribution has to be specified. Several analytic (usually approximate) solution of the dispersion equation with various momentum distributions can be found in $[10,13,70,14]$. A typical example of the numerical solution, which gives the unstable mode frequency in the full range of wave vectors is shown in Fig. 2 taken from [12]. The momentum distribution is of the form

$$
f(\mathbf{p}) \sim \frac{1}{\left(p_{T}^{2}+\sigma_{\perp}^{2}\right)^{3}} \mathrm{e}^{-\frac{p_{z}^{2}}{2 \sigma_{\|}^{2}}}
$$

where $p_{\perp} \equiv \sqrt{p_{x}^{2}+p_{y}^{2}}$. The mode is pure imaginary and $\gamma_{k} \equiv \operatorname{Im} \omega\left(k_{\perp}\right)$. The value of the coupling is $\alpha_{s} \equiv g^{2} / 4 \pi=0.3, \sigma_{\perp}=0.3 \mathrm{GeV}$ and the effective parton density is chosen to be $6 \mathrm{fm}^{-3}$. As seen, there is a finite interval of wave vectors for which the unstable modes exist.

The dispersion equation (6.5) corresponds to a simple configuration where the wave vector is parallel to the axis $x$ and it points to the direction of the momentum deficit while the chromoelectric field is parallel to the axis $z$ and it points to the momentum surplus. However, there are more general unstable modes which are not aligned along the symmetry axes of the momentum distribution of particles. The wave vectors $\mathbf{k}$ and chromoelectric fields $\mathbf{E}$ of these modes have non-vanishing components in the directions of the momentum deficit and momentum surplus, respectively, and $\mathbf{E}$ is no longer perpendicular to $\mathbf{k}$. Such unstable modes are discussed in [12]. A quite general analysis of the dispersion equation of anisotropic systems is given in [13, 70]. There is considered a class of momentum distributions which can be expressed as

$$
f(\mathbf{p})=f_{\text {iso }}\left(\sqrt{\mathbf{p}^{2}+\xi(\mathbf{n p})^{2}}\right)
$$



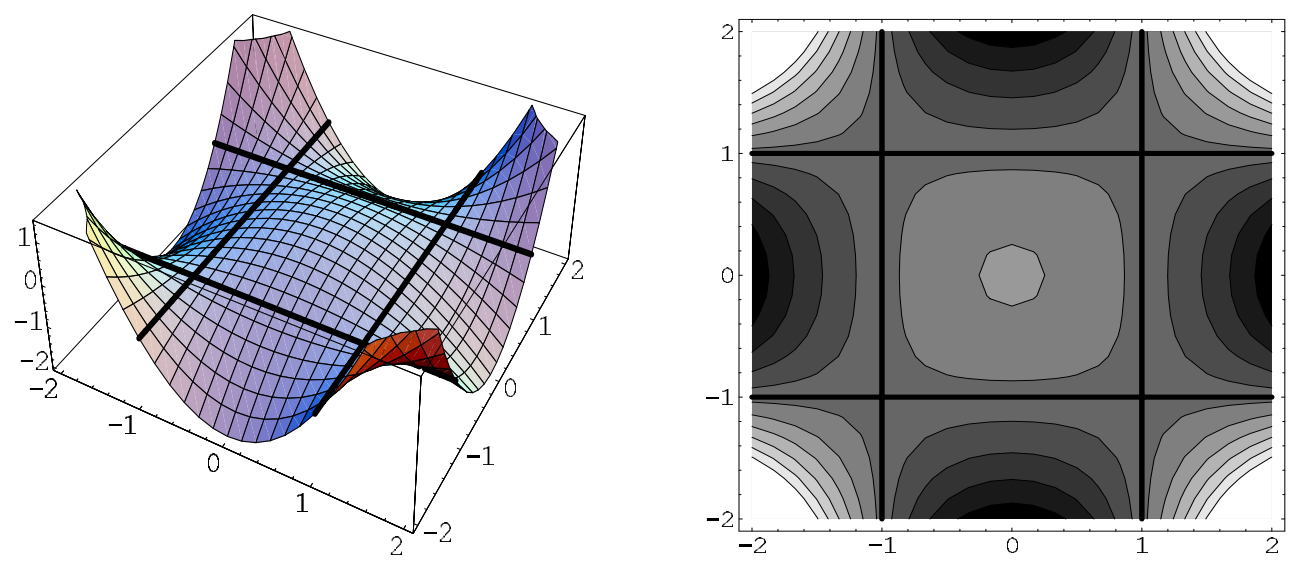

Figure 3: The effective potential of the unstable magnetic mode as a function of magnitude of two colour components of $\mathbf{A}^{a}$ belonging to the SU(2) gauge group. The figure is taken from [74].

where $f_{\text {iso }}(|\mathbf{p}|)$ is an arbitrary (isotropic) distribution, the unit vector $\mathbf{n}$ defines a preferred direction and the parameter $\xi \in(-1, \infty)$ controls the magnitude of anisotropy.

As explained above, the existence of the unstable gluonic modes is a generic feature of the anisotropic plasma - even a weak anisotropy generates the instability. In contrary, the quark modes seem to be always stable [71, 72]. Although, a general proof of the quark mode stability is lacking, the modes appear to be stable even for an extremely anisotropic parton momentum distribution as in the two-stream system [71] or as in the case of infinitely oblate distribution $(\xi=\infty)$ [72]. Presumably, the quark modes are always stable because their population is constrained by Pauli blocking [72].

\section{Isotropization and Abelianization}

When the instabilities grow the system becomes more isotropic because the Lorentz force changes the particle's momenta and the growing fields carry an extra momentum. To explain the mechanism I assume, as previously, that initially there is a momentum surplus in the $z$ direction. The fluctuating current flows in the $z$ direction with the wave vector pointing in the $x$ direction. Since the magnetic field has a $y$ component, the Lorentz force, which acts on partons flying along the $z$ axis, pushes the partons in the $x$ direction where there is a momentum deficit. Numerical simulations discussed in Sec. 10 show that growth of the instabilities is indeed accompanied with the system's fast isotropization.

The system isotropizes not only due to the effect of the Lorentz force but also due to the momentum carried by the growing field. When the magnetic and electric fields are oriented along the $y$ and $z$ axes, respectively, the Poynting vector points in the direction $x$ that is along the wave vector. Thus, the momentum carried by the fields is oriented in the direction of the momentum deficit of particles.

Unstable modes cannot grow to infinity and even in the electron-ion plasma there are several possible mechanisms which stop the instability growth [73]. The actual mechanism depends on the plasma state as well as on the external conditions. In the case of the quark-gluon plasma one suspects that non-Abelian non-linearities can play an important role here. An elegant argument [74] 
suggests that the non-linearities do not stabilize the unstable modes because the system spontaneously chooses an Abelian configuration in the course of instability development. Let me explain the idea.

In the Coulomb gauge the effective potential of the unstable configuration has the form

$$
V_{\mathrm{eff}}\left[\mathbf{A}^{a}\right]=-\mu^{2} \mathbf{A}^{a} \cdot \mathbf{A}^{a}+\frac{1}{4} g^{2} f^{a b c} f^{a d e}\left(\mathbf{A}^{b} \mathbf{A}^{d}\right)\left(\mathbf{A}^{c} \mathbf{A}^{e}\right)
$$

which is shown in Fig. 3 taken from [74]. The first term (with $\mu^{2}>0$ ) is responsible for the very existence of the instability. The second term, which comes from the Yang-Mills lagrangian, is of pure non-Abelian nature. The term appears to be positive and thus it counteracts the instability growth. However, the non-Abelian term vanishes when the potential $\mathbf{A}^{a}$ is effectively Abelian, and consequently, such a configuration corresponds to the steepest decrease of the effective potential. Thus, the system spontaneously abelianizes in the course of instability growth. In Sec. 10, where the results of numerical simulations are presented, the abelianization is further discussed.

\section{Hard-Loop Effective Action}

Knowledge of the gluon polarization tensor or, equivalently, the chromoelectric permittivity tensor is sufficient to discuss the system's stability and the dispersion relations of unstable modes. For more detailed dynamical studies the effective action of anisotropic quark-gluon plasma is needed. Such an action for a system, which is on average locally colour neutral, stationary and homogeneous, was derived and discussed in [75], see also [76]. The starting point was the effective action which describes an interaction of classical fields with currents induced by these fields in the plasma. The lagrangian density is quadratic in the gluon and quark fields and it equals

$$
\mathscr{L}_{2}(x)=-\int d^{4} y\left(\frac{1}{2} A_{\mu}^{a}(x) \Pi_{a b}^{\mu v}(x-y) A_{v}^{b}(y)+\bar{\Psi}(x) \Sigma(x-y) \Psi(y)\right) ;
$$

the Fourier transformed gluon polarization tensor $\Pi_{a b}^{\mu v}(k)$ and the quark self-energy $\Sigma(k)$ read

$$
\begin{aligned}
\Pi_{a b}^{\mu v}(k) & =\delta_{a b} \frac{g^{2}}{2} \int \frac{d^{3} p}{(2 \pi)^{3}} \frac{f(\mathbf{p})}{|\mathbf{p}|} \frac{(p \cdot k)\left(k^{\mu} p^{v}+p^{\mu} k^{v}\right)-k^{2} p^{\mu} p^{v}-(p \cdot k)^{2} g^{\mu v}}{(p \cdot k)^{2}} \\
\Sigma(k) & =g^{2} \frac{N_{c}^{2}-1}{8 N_{c}} \int \frac{d^{3} p}{(2 \pi)^{3}} \frac{\tilde{f}(\mathbf{p})}{|\mathbf{p}|} \frac{p \cdot \gamma}{p \cdot k}
\end{aligned}
$$

where $f(\mathbf{p})$ and $\tilde{f}(\mathbf{p})$ are the effective parton distribution functions defined as $f(\mathbf{p}) \equiv n(\mathbf{p})+\bar{n}(\mathbf{p})+$ $2 N_{c} n_{g}(\mathbf{p})$ and $\tilde{f}(\mathbf{p}) \equiv n(\mathbf{p})+\bar{n}(\mathbf{p})+2 n_{g}(\mathbf{p}) ; n(\mathbf{p}), \bar{n}(\mathbf{p})$ and $n_{g}(\mathbf{p})$ are, as already mentioned below Eq. (4.1), the distribution functions of quarks, antiquarks and gluons of single colour component in a homogeneous and stationary plasma which is locally and globally colourless; the spin and flavour are treated as parton internal degrees of freedom. The quarks and gluons are assumed to be massless. The polarization tensor (8.2) can be derived within the semiclassical transport theory $[69,13]$ or diagrammatically [69], following the formal rules of the Hard Thermal Loop approach. The quark self-energy (8.3) has been derived so far only diagrammatically $[69,77]$ but the derivation is also possible within the transport theory, as it has been done in [78] for the case of 
equilibrium plasma. The action (8.1) holds under the assumption that the field amplitude is much smaller than $T / g$ where $T$ denotes the characteristic momentum of (hard) partons.

Following Braaten and Pisarski [79], the lagrangian (8.1) was modified to comply with the requirement of gauge invariance. The final result, which is non-local but manifestly gauge invariant, is [75]

$$
\begin{aligned}
& \mathscr{L}_{\mathrm{HL}}(x)=\frac{g^{2}}{2} \int \frac{d^{3} p}{(2 \pi)^{3}} {\left[f(\mathbf{p}) F_{\mu v}^{a}(x)\left(\frac{p^{v} p^{\rho}}{(p \cdot D)^{2}}\right)_{a b} F_{\rho}{ }^{b \mu}(x)\right.} \\
&\left.+i \frac{N_{c}^{2}-1}{4 N_{c}} \tilde{f}(\mathbf{p}) \bar{\Psi}(x) \frac{p \cdot \gamma}{p \cdot D} \Psi(x)\right]
\end{aligned}
$$

where $F_{a}^{\mu v}$ is the strength tensor and $D$ denotes the covariant derivative. The effective action (8.4) generates $n$-point functions which obey the Ward-Takahashi identities. For the equilibrium plasma the action (8.4) is equivalent to that one derived in [80] and in the explicitly gauge invariant form in [79]. The equilibrium Hard Loop action was also found within the semiclassical kinetic theory $[78,81]$.

\section{Equations of motion}

Transport theory provides a natural framework to study temporal evolution of non-equilibrium systems and it has been applied to the quark-gluon plasma for a long time. The distribution functions of quarks $(Q)$, antiquarks $(\bar{Q})$, and gluons $(G)$, which are the $N_{c} \times N_{c}$ and $\left(N_{c}^{2}-1\right) \times\left(N_{c}^{2}-1\right)$ matrices, respectively, satisfy the transport equations of the form [82, 83]:

$$
\begin{array}{r}
p^{\mu} D_{\mu} Q(\mathbf{p}, x)+\frac{g}{2} p^{\mu}\left\{F_{\mu v}(x), \frac{\partial Q(\mathbf{p}, x)}{\partial p_{v}}\right\}=0, \\
p^{\mu} D_{\mu} \bar{Q}(\mathbf{p}, x)-\frac{g}{2} p^{\mu}\left\{F_{\mu v}(x), \frac{\partial \bar{Q}(\mathbf{p}, x)}{\partial p_{v}}\right\}=0, \\
p^{\mu} \mathscr{D}_{\mu} G(\mathbf{p}, x)+\frac{g}{2} p^{\mu}\left\{\mathscr{F}_{\mu v}(x), \frac{\partial G(\mathbf{p}, x)}{\partial p_{v}}\right\}=0,
\end{array}
$$

where $\{\ldots, \ldots\}$ denotes the anticommutator; the transport equation of (anti-)quarks is written down in the fundamental representation while that of gluons in the adjoint one. Since the instabilities of interest are very fast, much faster than the inter-parton collisions, the collision terms are neglected in Eqs. (9.1). The gauge field, which enters the transport equations (9.1), is generated self-consistently by the quarks and gluons. Thus, the transport equations (9.1) should be supplemented by the Yang-Mills equation

$$
D_{\mu} F^{\mu v}(x)=j^{v}(x)
$$

where the colour current is given as

$$
j^{\mu}(x)=-g \int \frac{d^{3} p}{(2 \pi)^{3}} \frac{p^{\mu}}{|\mathbf{p}|} \tau_{a}\left[\operatorname{Tr}\left[\tau_{a}(Q(\mathbf{p}, x)-\bar{Q}(\mathbf{p}, x))\right]+\operatorname{Tr}\left[T_{a} G(\mathbf{p}, x)\right]\right],
$$

with $\tau_{a}$ and $T_{a}$ being the $\mathrm{SU}\left(N_{c}\right)$ group generators in the fundamental and adjoint representation, respectively. There is a version of the equations $(9.1,9.2)$ where colour charges of partons are 
treated as a classical variable [84]. Then, the distribution functions depend not only on $x$ and $\mathbf{p}$ but on the colour variable as well.

When the equations $(9.1,9.2)$ are linearized around the state, which is stationary, homogeneous and locally colourless, the equations provide the Hard Loop dynamics encoded in the effective action (8.4). The equations are of particularly simple and elegant form when the quark $\delta Q(\mathbf{p}, x)$, antiquark $\delta \bar{Q}(\mathbf{p}, x)$ and gluon $\delta G(\mathbf{p}, x)$ deviations from the stationary state described by $Q_{0}^{j j}(\mathbf{p})=$ $\delta^{i j} n(\mathbf{p}), \bar{Q}_{0}^{i j}(\mathbf{p})=\delta^{i j} \bar{n}(\mathbf{p})$, and $G_{0}^{a b}(\mathbf{p})=\delta^{a b} n_{g}(\mathbf{p})$ are parameterised by the field $W^{\mu}(\mathbf{v}, x)$ through the relations

$$
\begin{gathered}
\delta Q(\mathbf{p}, x)=g \frac{\partial n(\mathbf{p})}{\partial p^{\mu}} W^{\mu}(\mathbf{v}, x), \quad \delta \bar{Q}(\mathbf{p}, x)=-g \frac{\partial \bar{n}(\mathbf{p})}{\partial p^{\mu}} W^{\mu}(\mathbf{v}, x), \\
\delta G(\mathbf{p}, x)=g \frac{\partial n_{g}(\mathbf{p})}{\partial p^{\mu}} T_{a} \operatorname{Tr}\left[\tau_{a} W^{\mu}(\mathbf{v}, x)\right],
\end{gathered}
$$

where $\mathbf{v} \equiv \mathbf{p} /|\mathbf{p}|$. Then, instead of the three transport equations (9.1) one has one equation

$$
v_{\mu} D^{\mu} W^{v}(\mathbf{v}, x)=-v_{\rho} F^{\rho v}(x)
$$

while the Yang-Mills equation (9.2) reads

$$
D_{\mu} F^{\mu v}(x)=j^{v}(x)=-g^{2} \int \frac{d^{3} p}{(2 \pi)^{3}} \frac{p^{v}}{|\mathbf{p}|} \frac{\partial f(\mathbf{p})}{\partial p^{\rho}} W^{\rho}(\mathbf{v}, x),
$$

where $v^{\mu}=(1, \mathbf{v})$ and, as previously, $f(\mathbf{p}) \equiv n(\mathbf{p})+\bar{n}(\mathbf{p})+2 N_{c} n_{g}(\mathbf{p})$. In contrast to the effective action $(8.4)$, the equations $(9.4,9.5)$ are local in coordinate space. Therefore, the transport equation (9.4) combined with Eq. (9.5) is often called local representation of the Hard Loop dynamics. The equations $(9.4,9.5)$, which for the isotropic equilibrium plasma were first given in [67], are used in the numerical simulations $[15,85,86,87]$ discussed in the next section.

Recently the fluid equations, which are applicable to short-time scale colour phenomena in the quark-gluon plasma, have been derived [88] from the kinetic equations (9.1). The quantities, which enter the equations, like the hydrodynamic velocity or pressure are gauge dependent matrices in the colour space. The linearized fluid equations were solved analytically but the chromo-hydrodynamic approach is rather designed for numerical studies of the dynamics of the unstable quark-gluon plasma.

\section{Numerical simulations}

Temporal evolution of the anisotropic quark-gluon plasma has been recently studied by means of numerical simulations $[15,16,85,86,87,89,91,92]$. The simulations, which have been performed in two very different dynamical schemes by three groups of authors, are of crucial importance as they convincingly demonstrate a key role of the instabilities in the evolution of anisotropic quark-gluon plasma.

The dynamics governed by the Hard Loop action (8.4) and described by the equations $(9.4,9.5)$ has been simulated in $[15,85,86,87,92]$. These simulations provide fully a reliable information on the field dynamics provided the potential's amplitude is not too large: $A_{a}^{\mu} \ll T / g$ where $T$ 


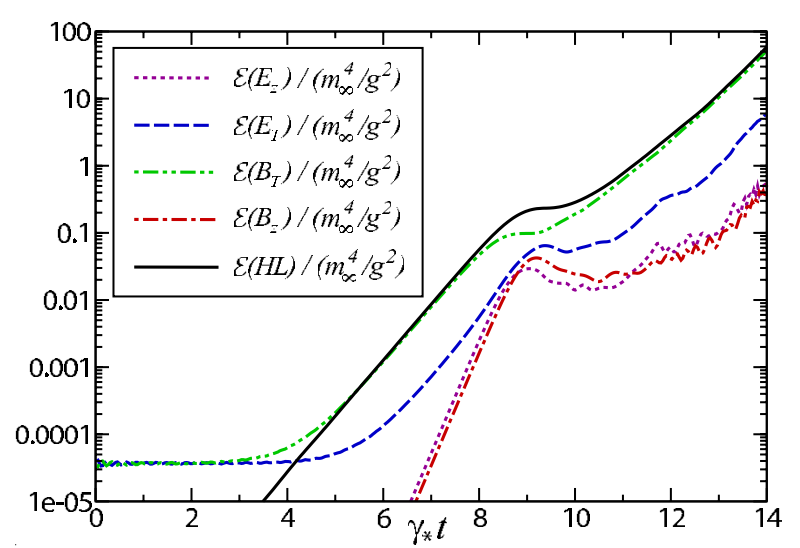

Figure 4: Time evolution of the (scaled) energy density (split into various electric and magnetic components) which is carried by the chromodynamic field. The simulation is $1+1$ dimensional and the gauge group is $\mathrm{SU}(2)$. The parton momentum distribution is squeezed along the $z$ axis. The solid line corresponds to the total energy transferred from the particles. The figure is taken from [15].

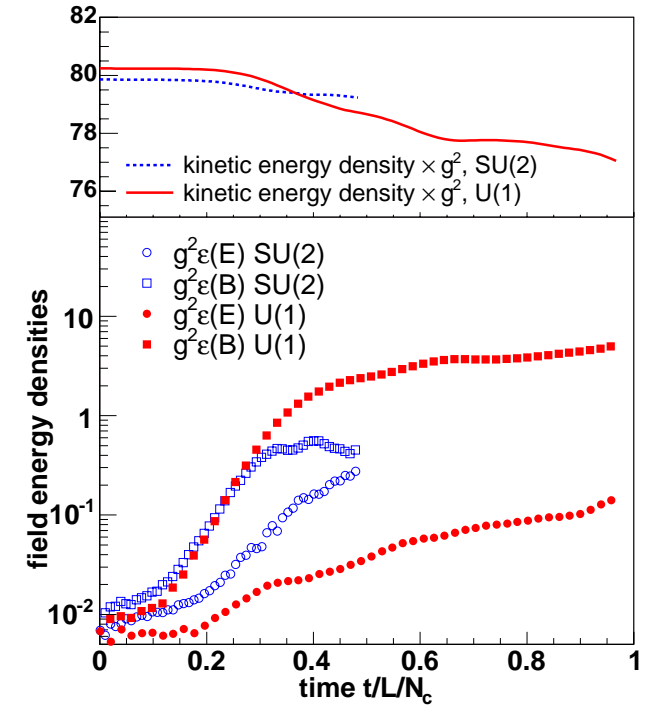

Figure 5: Time evolution of the kinetic energy of particles (upper panel) and of the energy of electric and magnetic fields (lower panel) in $\mathrm{GeV} / \mathrm{fm}^{3}$ for the $\mathrm{U}(1)$ and $\mathrm{SU}(2)$ gauge groups. The figure is taken from [16].

is the characteristic momentum of (hard) partons. Since the equations $(9.4,9.5)$ describe small deviations from the stationary homogeneous state, only a small fraction of the particles is influenced by the growing chromodynamic fields. Therefore, the (hard) particles effectively play a role of the stationary (anisotropic) background. In the simulations [16, 89, 91] the classical version of the equations $(9.1,9.2)$ is used. The quark-gluon plasma is treated as a completely classical system: partons, which carry classical colour charges, interact with the self-consistently generated classical chromodynamic field.

The simulations $[15,16]$ have been effectively performed in $1+1$ dimensions as the chromodynamic potentials depend on time and one space variable. The calculations $[85,86]$ represent full 1+3 dimensional dynamics. In most cases the SU(2) gauge group was studied but some SU(3) results, which are qualitatively very similar to $\mathrm{SU}(2)$ ones, are given in [86].

The techniques of discretization used in the simulations [15, 16, 85, 86, 87, 89, 91] are rather different while the initial conditions are quite similar. The initial field amplitudes are distributed according to the Gaussian white noise and the momentum distribution of (hard) partons is strongly anisotropic. For example, in the classical simulation [16] the initial parton momentum distribution is chosen as

$$
f(\mathbf{p}) \sim \delta\left(p_{x}\right) \mathrm{e}^{-\frac{\sqrt{p_{y}^{2}+p_{z}^{2}}}{p_{\text {hard }}}}
$$

with $p_{\text {hard }}=10 \mathrm{GeV}$. The results are actually insensitive to the specific form of the momentum distribution. If the parton distribution function is written in the form (6.6), the results are shown [15, 13 ] to depend only on two parameters: $\xi$ and the Debye mass $m_{\mathrm{D}}$ of the corresponding isotropic 


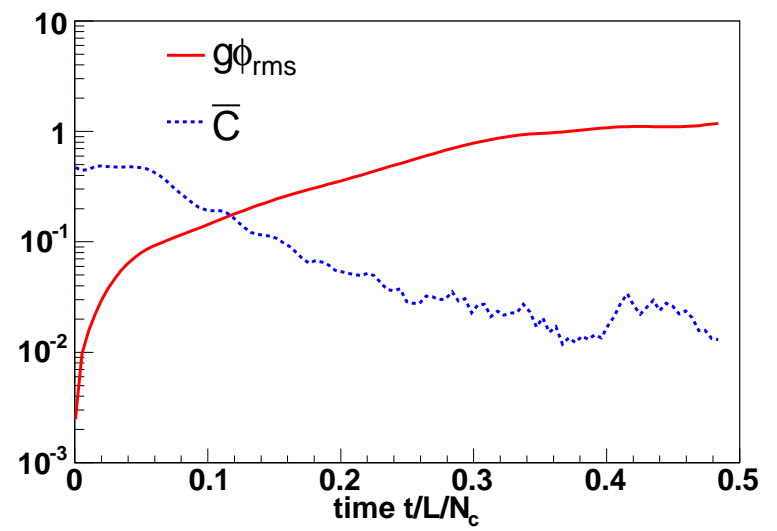

Figure 6: Temporal evolution of the functionals $\bar{C}$ and $\phi_{\mathrm{rms}}$ measured in GeV. The figure is taken from [16].

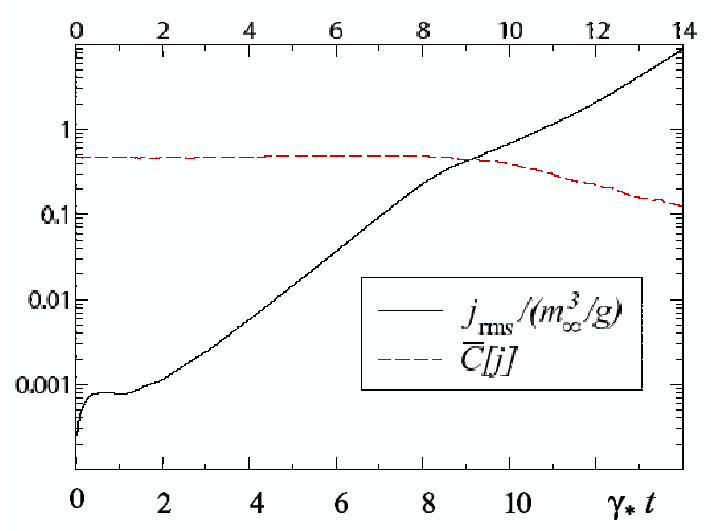

Figure 7: Temporal evolution of the (scaled) functionals $\bar{C}$ and $j_{\text {rms. }}$. The figure is taken from [15].

system i.e.

$$
m_{\mathrm{D}}^{2}=-\frac{g^{2}}{4 \pi^{2}} \int_{0}^{\infty} d p p^{2} \frac{\partial f_{\text {iso }}(p)}{\partial p} .
$$

In Fig. 4, taken from [15], the results of the Hard-Loop simulation performed in $1+1$ dimensions are shown. One observes exponential growth of the energy density stored in fields and the energy density is dominated, as expected, by the magnetic field which is transverse to the direction of the momentum deficit. The growth rate of the energy density appears to be equal to the growth rate $\gamma^{*}$ of the fastest unstable mode. Fig. 5, which is taken from [16], shows results of the classical simulation on the $1+1$ dimensional lattice of physical size $L=40 \mathrm{fm}$. As in Fig. 4 , the amount of field energy, which is initially much smaller than the kinetic energy of all particles, grows exponentially and the magnetic contribution dominates.

The Abelian (U(1)) and non-Abelian (SU(2)) results of the 1+1 dimensional simulation presented in Fig. 5 are remarkably similar to each other. The abelianization, explained in Sec. 7, appears to be very efficient in 1+1 dimensions, as shown in Figs. 6, 7, taken from [16] and [15], respectively. The authors of [16] analysed the functionals

$$
\phi_{\mathrm{rms}} \equiv \sqrt{2 \int_{0}^{L} \frac{d x}{L} \operatorname{Tr}\left[A_{y}^{2}+A_{z}^{2}\right]}, \quad \bar{C} \equiv \int_{0}^{L} \frac{d x}{L} \frac{\sqrt{\operatorname{Tr}\left[\left(i\left[A_{y}, A_{z}\right]\right)^{2}\right]}}{\operatorname{Tr}\left[A_{y}^{2}+A_{z}^{2}\right]},
$$

which were introduced in [74]. The quantities $j_{\mathrm{rms}}$ and $\bar{C}$, studied in [15] and shown in Fig. 7, are fully analogous to $\phi_{\mathrm{rms}}$ and $\bar{C}$ defined by Eq. (10.2) but the components of chromodynamic potential are replaced by the respective components of colour current. As seen in Figs. 6, 7, the field (current) commutator decreases in time although the magnitude of field (current), as quantified by $\phi_{\mathrm{rms}}\left(j_{\mathrm{rms}}\right)$, grows.

It is worth mentioning that the functionals (10.2) defined through the gauge potentials are gauge invariant provided the potentials depend only of one time and one space variables and the gauge transformations preserve this property. Thus, the functionals (10.2) are well suited for $1+1$ dimensional simulations. However, the functionals (10.2) are not gauge invariant under general $1+3$ dimensional gauge transformations. When the potential components are replaced by the 


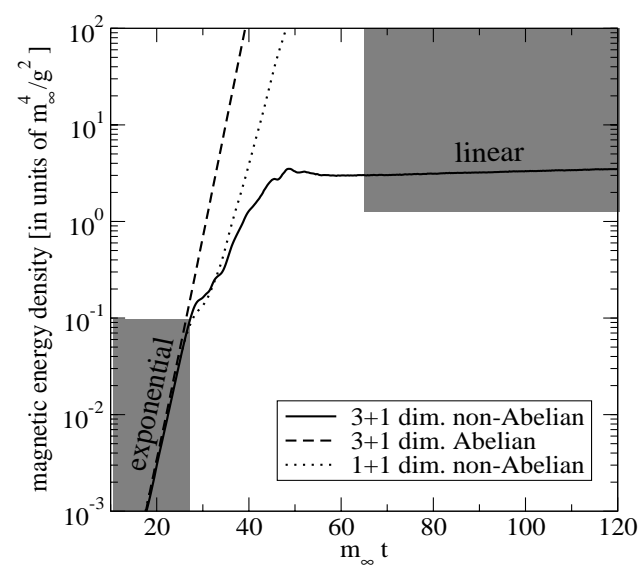

Figure 8: Time evolution of the (scaled) chromomagnetic energy density in the $1+3$ dimensional simulation. The Abelian result and that of $1+1$ dimensions are also shown. The figure is taken from [85].

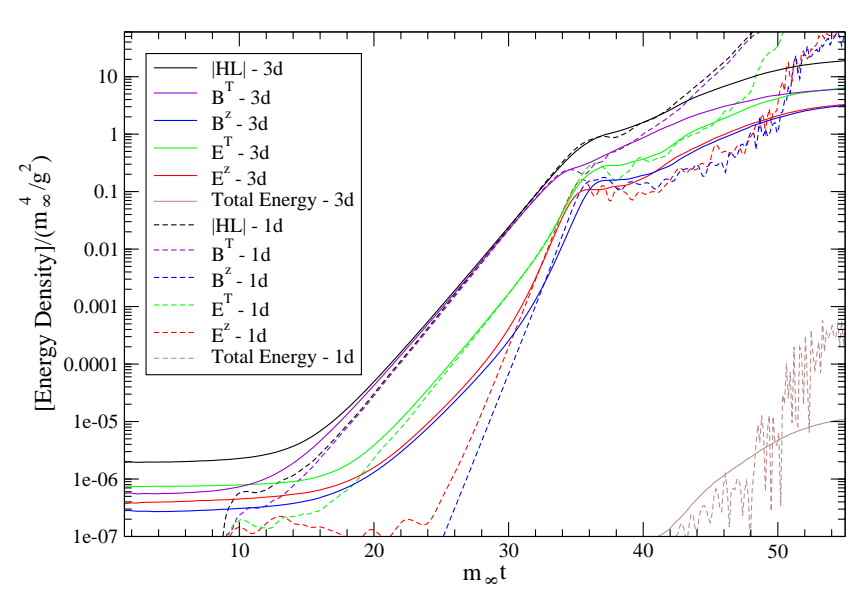

Figure 9: Time evolution of the (scaled) energy density (split into various electric and magnetic components) of the chromodynamic field in the $1+1$ and $1+3$ simulations. ' $\mathrm{HL}$ ' denotes the total energy contributed by hard particles. The figure is taken from [86].

respective current components, as proposed in [15], the functionals are gauge invariant not only under $1+1$ but also under $1+3$ dimensional transformations.

The results of the $1+3$ dimensional simulations $[85,86]$ appear to be qualitatively different from those of $1+1$ dimensions. As seen in Figs. 8, 9, taken from [85] and [86], respectively, the growth of the field energy density is exponential only for some time, and then the growth becomes approximately linear. It appears that the regime changes when the field's amplitude is of order $\mathrm{k} / \mathrm{g}$ where $k$ is the characteristic field wave vector. Then, the non-Abelian effects start to be important. Indeed, Fig. 10, which is taken from [85], demonstrates that the abelianization is efficient in the $1+3$ dimensional simulations $[85,86]$ only for a finite interval of time. The commutator $C$ shown in Fig. 10, which is a natural generalization of the $1+1$ dimensional commutator defined by Eq. (10.2) with the current components instead of the potential ones, first decreases but after some time it starts to grow and returns to its initial value.

The regime of linear growth of the magnetic energy, shown in Figs. 8, 9 was studied numerically in [87]. It was found that when the exponential growth of the magnetic energy ends, the long-wavelength modes associated with the instability stop growing, but that they cascade energy towards the ultraviolet in the form of plasmon excitations and a quasi-stationary state with the power law distribution $k^{-2}$ of the plasmon mode population appears. The phenomenon was argued [87] to be very similar to the Kolmogorov wave turbulence where the long-wavelength modes transfer their energy without dissipation to the shorter and shorter ones.

A different picture of the nonAbelian regime emerges from the classical simulation [91] where the system with strong momentum anisotropy was studied. When the field strength is high enough, the energy drained by the Weibel-like plasma instability from the particles does not build up exponentially in magnetic fields but instead returns isotropically to the ultraviolet not via the quasistationary process, as argued in [87], but via a rapid avalanche. 
The effect of isotropization due to the action of the Lorentz force is nicely seen in the $1+1 \mathrm{di}-$ mensional classical simulation [16]. In Fig. 11, which is taken from [16], there are shown diagonal components of the energy-momentum tensor

$$
T^{\mu v}=\int \frac{d^{3} p}{(2 \pi)^{3}} \frac{p^{\mu} p^{v}}{E_{p}} f(\mathbf{p})
$$

The initial momentum distribution is given by Eq. (10.1), and consequently $T^{x x}=0$ at $t=0$. As seen in Fig. 11, $T^{x x}$ exponentially grows. However, a full isotropy, which requires $T^{x x}=\left(T^{y y}+\right.$ $\left.T^{z z}\right) / 2$, is not achieved.

The numerical studies discussed so far deal with the quark-gluon system of constant volume. A very elegant formulation of the Hard Loop dynamics of the system, which experiences the boost invariant expansion in one direction, is given in [92]. In agreement with the earlier expectations $[12,14]$, the expansion is shown both numerically and analytically [92] to slow down growth of instabilities even when the initial state is highly anisotropic. The field amplitude does not grow exponentially with time but rather as the exponent of $\sqrt{t}$. The effect of expansion requires further quantitative analysis, as the instabilities might occur irrelevant for heavy-ion collisions, if they are not fast enough to cope with the system's expansion.

An attempt to study an unstable parton system in the conditions close to those, which are realized in relativistic heavy-ion collisions, was undertaken in $[93,94,95]$. The system was described in terms of the Colour Glass Condensate approach [48] where small $x$ partons of large occupation numbers, which dominate the wave functions of incoming nuclei, are treated as classical YangMills fields. Hard modes of the classical fields play the role of particles. The instabilities, identified as the Weibel modes, appear to be generated when the system of Yang-Mills fields expands into vacuum.

\section{Outlook and Final Remarks}

One wonders whether the presence of the instabilities at the early stage of relativistic heavy-ion collisions is experimentally observable. The accelerated equilibration is obviously very important though it is only an indirect signal. It has been suggested $[96,97]$ that strong chromomagnetic fields generated by the instabilities can lead to a specific pattern of jet's deflections. This promising proposal, however, requires further studies.

Another idea has been formulated in [98]. The quark-gluon plasma, which is initially anisotropic, is isotropized fast due to the magnetic instabilities. Such a non-equilibrium plasma manifests, as recently observed [11], an approximate hydrodynamic behaviour even before the equilibrium is reached. The point is that the structure of the ideal fluid energy-momentum tensor i.e. $T^{\mu v}=$ $(\varepsilon+p) u^{\mu} u^{v}-p g^{\mu v}$, where $\varepsilon, p$ and $u^{\mu}$ is the energy density, pressure and hydrodynamic velocity, respectively, holds for an arbitrary but isotropic momentum distribution. $\varepsilon$ and $p$ are then not the energy density and pressure but the moments of the distribution function which are equal the energy density and pressure in the equilibrium limit. Since the tensor $T^{\mu v}$ always obeys the continuity equation $\partial_{\mu} T^{\mu v}=0$, one gets an analogue of the Euler equation. However, due to the lack of thermodynamic equilibrium there is no entropy conservation and the equation of state is missing. 


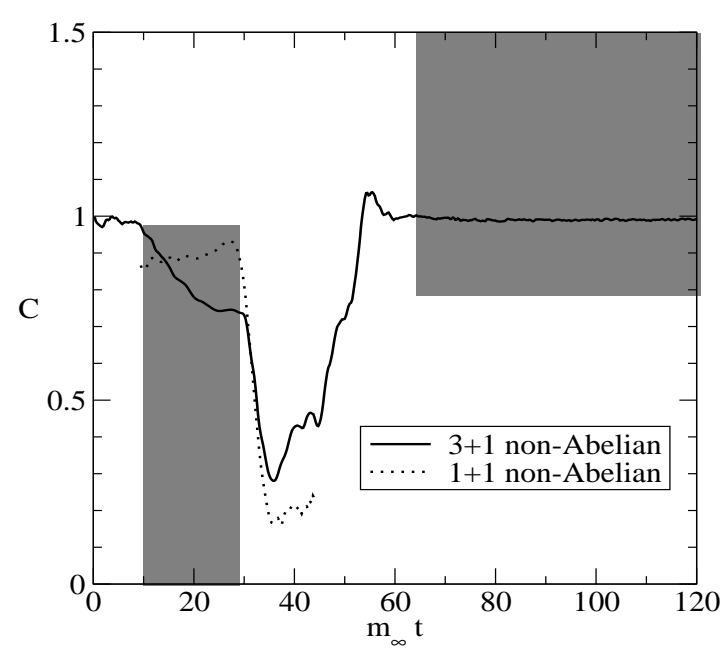

Figure 10: Temporal evolution of the field commutator quantified by $C$. The figure is taken from [85].

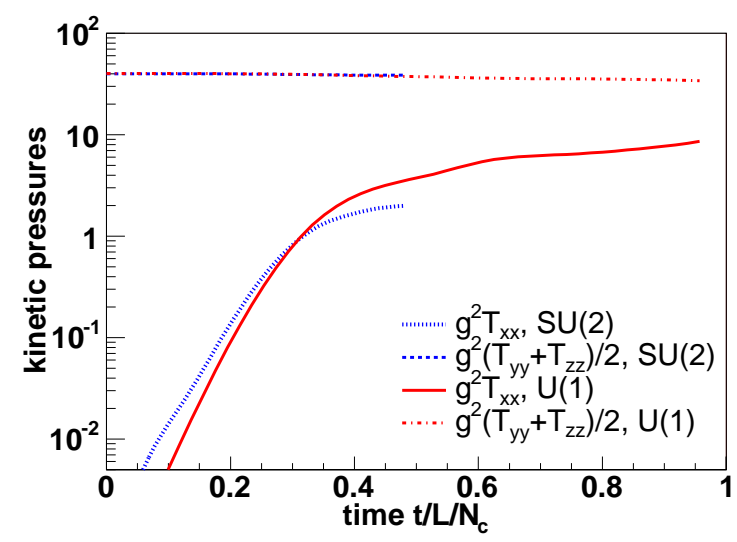

Figure 11: Temporal evolution of the energymomentum tensor components $T^{x x}$ and $\left(T^{y y}+T^{z z}\right) / 2$. The Abelian and non-Abelian results are shown. The figure is taken from [16].

The azimuthal fluctuations have been argued [98] to distinguish the approximate hydrodynamics - characteristic for the instabilities driven isotropization - from the real hydrodynamics describing a system which is in a local thermodynamic equilibrium, as advocated by proponents of the strongly coupled plasma [5]. Non-equilibrium fluctuations are usually significantly larger than the equilibrium fluctuations of the same quantity. A specific example of such a situation is given in Sec. 4 where the current fluctuations in the anisotropic system are discussed. Thus, one expects that the (computable) fluctuations of $v_{2}$ produced in the course of real hydrodynamic evolution are significantly smaller than those generated in the non-equilibrium quark-gluon plasma which is merely isotropic. It should be stressed here that the elliptic flow is generated in the collision relatively early stage when there is a large configuration-space asymmetry of the colliding system. Since a measurement of $v_{2}$ fluctuations is rather difficult, it was also argued [98] that an integral measurement of the azimuthal fluctuations can help as well to distinguish the equilibrium from non-equilibrium fluctuations. Further suggestions of detectable signals of the instabilities are very much needed. However, an experimental verification will certainly require much better theoretical understanding of the equilibration process.

Although an impressive progress has been achieved, the numerical simulations are still quite far from a real situation met in relativistic heavy-ion collisions. Complete $1+3$ dimensional simulations are needed, as the results of $[85,86]$ show that the dimensionality crucially matters. The system expansion needs to be incorporated. The effect of back reaction of fields on the particles is fully included only in the classical simulations $[16,91,93,94,95]$. The effect is difficult to study in quantum field approaches as it goes beyond the Hard Loop physics which has appeared very rich and complex $[85,86]$. An attempt to go beyond the Hard Loop Approximation was been undertaken in [99] where the higher order terms of the effective potential of the anisotropic system were found. Since these terms can be negative, the instability is then driven not only by the negative quadratic term but by the higher order terms as well. 
The parton-parton collisions is another physically important effect which goes beyond the Hard Loop Approximation. Recently, the effect has been modelled [100] using the kinetic equations with the collision terms of the so-called BGK form (similar to the well-known Relaxation Time Approximation). The collisions have been shown to slow down growth of the unstable modes, and there is an upper limit on the collisional frequency beyond which no instabilities exist.

The coupling constant is assumed to be small in all studies of the unstable parton systems. This is certainly a severe limitation as the phenomenology of heavy-ion collisions suggests that the quark-gluon plasma manifests very small viscosity characteristic for strongly coupled systems [5]. However, it has been recently argued $[101,102]$ that an anomalously small viscosity of the quarkgluon system can arise from interactions with turbulent colour fields dynamically generated by the instabilities. Therefore, it might well be that the scenario of instabilities driven equilibration does not only solve the problem of fast thermalization but other puzzling features of the quark-gluon plasma as well.

In summary, the magnetic instabilities provide a plausible explanation of the surprisingly short equilibration time observed in relativistic heavy-ion collisions. The explanation does not require a strong coupling of the quark-gluon plasma. Fast isotropization of the system is a distinctive feature of the instabilities driven equilibration. Two signals of the instabilities have been suggested but quantitative predictions are lacking. New ideas are certainly needed. In spite of the impressive progress, which has been achieved recently, a theoretical description of the unstable quark-gluon plasma requires further improvements.

\section{References}

[1] St. Mrówczyński, Acta Phys. Polon. B 37 (2006) 427.

[2] U. W. Heinz, arXiv:nucl-th/0504011.

[3] F. Retiere, J. Phys. G 30 (2004) S827.

[4] U. W. Heinz, AIP Conf. Proc. 739 (2005) 163.

[5] E. Shuryak, J. Phys. G 30 (2004) S1221.

[6] H. Sorge, Phys. Rev. Lett. 82 (1999) 2048.

[7] R. Baier, A. H. Mueller, D. Schiff and D. T. Son, Phys. Lett. B 502 (2001) 51.

[8] R. Baier, A. H. Mueller, D. Schiff and D. T. Son, Phys. Lett. B 539 (2002) 46.

[9] P. Arnold, D. T. Son and L. G. Yaffe, Phys. Rev. D 59 (1999) 105020.

[10] St. Mrówczyński, Phys. Rev. C 49 (1994) 2191.

[11] P. Arnold, J. Lenaghan, G. D. Moore and L. G. Yaffe, Phys. Rev. Lett. 94 (2005) 072302.

[12] J. Randrup and St. Mrówczyński, Phys. Rev. C 68 (2003) 034909.

[13] P. Romatschke and M. Strickland, Phys. Rev. D 68 (2003) 036004.

[14] P. Arnold, J. Lenaghan and G. D. Moore, JHEP 0308 (2003) 002.

[15] A. Rebhan, P. Romatschke and M. Strickland, Phys. Rev. Lett. 94 (2005) 102303.

[16] A. Dumitru and Y. Nara, Phys. Lett. B 621 (2005) 89. 
[17] G. Baym, Phys. Lett. B 138 (1984) 18.

[18] S. Chakraborty and D. Syam, Lett. Nuovo Cim. 41 (1984) 381.

[19] K. Kajantie and T. Matsui, Phys. Lett. B 164 (1985) 373.

[20] R. C. Hwa and K. Kajantie, Phys. Rev. Lett. 56 (1986) 696.

[21] D. H. Boal, Phys. Rev. C 33 (1986) 2206.

[22] K. J. Eskola, K. Kajantie and J. Lindfors, Phys. Lett. B 214 (1988) 613.

[23] B. Banerjee, R. S. Bhalerao and V. Ravishankar, Phys. Lett. B 224 (1989) 16.

[24] K. Geiger and B. Muller, Nucl. Phys. B 369 (1992) 600.

[25] K. Geiger, Phys. Rev. D 46 (1992) 4965.

[26] K. Geiger, Phys. Rev. D 46 (1992) 4986.

[27] K. Geiger, Phys. Rept. 258 (1995) 237.

[28] X. N. Wang, Phys. Rept. 280 (1997) 287.

[29] E. V. Shuryak, Phys. Rev. Lett. 68 (1992) 3270.

[30] T. S. Biro, E. van Doorn, B. Muller, M. H. Thoma and X. N. Wang, Phys. Rev. C 48 (1993) 1275.

[31] J. Alam, B. Sinha and S. Raha, Phys. Rev. Lett. 73 (1994) 1895.

[32] H. Heiselberg and X. N. Wang, Phys. Rev. C 53 (1996) 1892.

[33] H. Heiselberg and X. N. Wang, Nucl. Phys. B 462 (1996) 389.

[34] L. Xiong and E. V. Shuryak, Phys. Rev. C 49 (1994) 2203.

[35] S. M. H. Wong, Nucl. Phys. A 607 (1996) 442.

[36] S. M. H. Wong, Phys. Rev. C 54 (1996) 2588.

[37] S. M. H. Wong, Phys. Rev. C 56 (1997) 1075.

[38] Z. Xu and C. Greiner, Phys. Rev. C 71 (2005) 064901.

[39] X. M. Xu, Y. Sun, A. Q. Chen and L. Zheng, Nucl. Phys. A 744 (2004) 347.

[40] S. M. H. Wong, arXiv:hep-ph/0404222.

[41] T. S. Biro, C. Gong, B. Muller and A. Trayanov, Int. J. Mod. Phys. C 5 (1994) 113.

[42] S. Sengupta, P. K. Kaw and J. C. Parikh, Phys. Lett. B 446 (1999) 104.

[43] V. M. Bannur, Phys. Rev. C 72 (2005) 024904.

[44] F. Cooper, E. Mottola and G. C. Nayak, Phys. Lett. B 555 (2003) 181.

[45] R. S. Bhalerao and G. C. Nayak, Phys. Rev. C 61 (2000) 054907.

[46] G. C. Nayak, A. Dumitru, L. D. McLerran and W. Greiner, Nucl. Phys. A 687 (2001) 457.

[47] G. R. Shin and B. Muller, J. Phys. G 29 (2003) 2485.

[48] E. Iancu and R. Venugopalan, in Quark-Gluon Plasma 3, edited by R.C. Hwa and X.N. Wang (World Scientific, Singapore, 2004).

[49] J. Serreau and D. Schiff, JHEP 0111 (2001) 039. 
[50] D. Bodeker, JHEP 0510 (2005) 092.

[51] A. H. Mueller, A. I. Shoshi and S. M. H. Wong, Phys. Lett. B 632 (2006) 257.

[52] A. Bialas, Phys. Lett. B 466 (1999) 301.

[53] W. Florkowski, Acta Phys. Polon. B 35 (2004) 799.

[54] D. Kharzeev and K. Tuchin, Nucl. Phys. A 753 (2005) 316.

[55] Y. V. Kovchegov, Nucl. Phys. A 762 (2005) 298.

[56] Y. V. Kovchegov, Nucl. Phys. A 764 (2006) 476.

[57] N.A. Krall and A.W. Trivelpiece, Principles of Plasma Physics (McGraw-Hill, New York, 1973).

[58] U. W. Heinz, Nucl. Phys. A 418 (1984) 603C.

[59] Y. E. Pokrovsky and A. V. Selikhov, JETP Lett. 47 (1988) 12 [Pisma Zh. Eksp. Teor. Fiz. 47 (1988) $11]$.

[60] Y. E. Pokrovsky and A. V. Selikhov, Sov. J. Nucl. Phys. 52, 146 (1990) [Yad. Fiz. 52, 229 (1990)].

[61] Y. E. Pokrovsky and A. V. Selikhov, Sov. J. Nucl. Phys. 52 (1990) 385 [Yad. Fiz. 52 (1990) 605].

[62] St. Mrówczyński, Phys. Lett. B 214 (1988) 587.

[63] O. P. Pavlenko, Sov. J. Nucl. Phys. 54 (1991) 884 [Yad. Fiz. 54 (1991) 1448].

[64] O. P. Pavlenko, Sov. J. Nucl. Phys. 55 (1992) 1243 [Yad. Fiz. 55 (1992) 2239].

[65] St. Mrówczyński, Phys. Lett. B 314 (1993) 118.

[66] E.S. Weibel, Phys. Rev. Lett. 2 (1959) 83.

[67] J. P. Blaizot and E. Iancu, Phys. Rept. 359 (2002) 355.

[68] St. Mrówczyński, Phys. Lett. B 393 (1997) 26.

[69] St. Mrówczyński and M. H. Thoma, Phys. Rev. D 62 (2000) 036011.

[70] P. Romatschke and M. Strickland, Phys. Rev. D 70 (2004) 116006.

[71] St. Mrówczyński, Phys. Rev. D 65 (2002) 117501.

[72] B. Schenke and M. Strickland, Phys. Rev. D 74 (2006) 065004.

[73] T. N. Kato, Phys. Plasmas 12 (2005) 080705.

[74] P. Arnold and J. Lenaghan, Phys. Rev. D 70 (2004) 114007.

[75] St. Mrówczyński, A. Rebhan and M. Strickland, Phys. Rev. D 70 (2004) 025004.

[76] R. D. Pisarski, arXiv:hep-ph/9710370.

[77] P. Arnold, G. D. Moore and L. G. Yaffe, JHEP 0301 (2003) 039.

[78] J. P. Blaizot and E. Iancu, Nucl. Phys. B 417608 (1994) 608.

[79] E. Braaten and R. D. Pisarski, Phys. Rev. D 45 (1992) 1827.

[80] J. C. Taylor and S. M. H. Wong, Nucl. Phys. B 346 (1990) 115.

[81] P. F. Kelly, Q. Liu, C. Lucchesi and C. Manuel, Phys. Rev. D 50 (1994) 4209.

[82] H. T. Elze and U. W. Heinz, Phys. Rept. 183 (1989) 81. 
[83] St. Mrówczyński, Phys. Rev. D 39 (1989) 1940.

[84] U. W. Heinz, Annals Phys. 161 (1985) 48.

[85] P. Arnold, G. D. Moore and L. G. Yaffe, Phys. Rev. D 72 (2005) 054003.

[86] A. Rebhan, P. Romatschke and M. Strickland, JHEP 0509 (2005) 041.

[87] P. Arnold and G. D. Moore, Phys. Rev. D 73 (2006) 025006.

[88] C. Manuel and St. Mrówczyński, Phys. Rev. D 74 (2006) 105003.

[89] A. Dumitru and Y. Nara, Eur. Phys. J. A 29 (2006) 65.

[90] P. Arnold and G. D. Moore, Phys. Rev. D 73 (2006) 025013.

[91] A. Dumitru, Y. Nara and M. Strickland, arXiv:hep-ph/0604149.

[92] P. Romatschke and A. Rebhan, arXiv:hep-ph/0605064.

[93] P. Romatschke and R. Venugopalan, Phys. Rev. Lett. 96 (2006) 062302.

[94] P. Romatschke and R. Venugopalan, Eur. Phys. J. A 29 (2006) 71

[95] P. Romatschke and R. Venugopalan, Phys. Rev. D 74 (2006) 045011.

[96] M. Strickland, private communication.

[97] B. Müller, Nucl. Phys. A 774 (2006) 433.

[98] St. Mrówczyński, J. Phys. Conf. Ser. 27 (2005) 204.

[99] C. Manuel and St. Mrówczyński, Phys. Rev. D 72 (2005) 034005.

[100] B. Schenke, M. Strickland, C. Greiner and M. H. Thoma, Phys. Rev. D 73 (2006) 125004.

[101] M. Asakawa, S. A. Bass and B. Muller, Phys. Rev. Lett. 96 (2006) 252301.

[102] M. Asakawa, S. A. Bass and B. Muller, arXiv:hep-ph/0608270. 